\title{
Ferrofluid Actuated Thermal Overload Relay
}

\author{
Badrinarayanan Rajagopalan, Mugundhan Hayagrivan, Mahesh Praveenkumar
}

Department of Electrical and Electronics Engineering, Panimalar Engineering College, Anna University, Chennai, India. Email: aswathnaray@gmail.com

Received September $26^{\text {th }}$, 2011; revised October $26^{\text {th }}, 2011$; accepted November $4^{\text {th }}, 2011$

\begin{abstract}
Thermal overload relays are economic electromechanical protection devices which offers reliable protection for electric motors in the event of overload or phase failure. Presently there are two types of overload relays which depend on the temperature characteristics of metals to provide protection by tripping the circuit. These relays lack accuracy as they do not activate the trip circuit at any exact specified temperature. In this paper we introduce a new form of thermal overload relay actuated by ferrofluid. The ferrofluid has a very accurate transition temperature known as curie temperature. It acts as a ferromagnetic material below the curie temperature and loses the property of ferromagnetism above this temperature. By using this property of the fluid we propose an alternative method for more accurate operation under overload condition. This relay finds application in the protection system of electrical machines. Thus, in this paper we present a novel and simple technique for protection against thermal overloading using ferrofluid.
\end{abstract}

Keywords: Ferrofluid; Overload Relay; Thermal Protection; Motor Protection

\section{Introduction}

A relay is an electrically operated switch which is used to control a circuit by a low-power signal with complete electrical isolation between control and controlled circuits. Thermal overload relays are designed to cut the power by opening the circuit when the motor draws too much current from the supply for an extended time causing temperature rise. Presently there are two types of thermal overload relays (Figure 1) in which one type has a bi-metallic strip [1] which deflects as the temperature rises until it causes the device to trip and open the circuit. A second type of thermal overload relay uses a eutectic alloy to retain a spring-loaded contact. When too much current passes through the heating element for a long time, the alloy melts and the spring releases the contact, opening the circuit.

Ferrofluid are smart fluids which has the fluid properties of a liquid and the magnetic properties of a solid. We use the thermal property of this fluid in the construction of thermal overload relay. The temperature rise in the

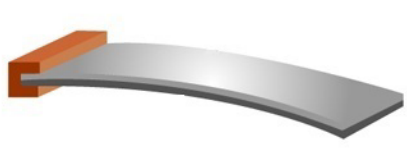

Bimetallic Strip

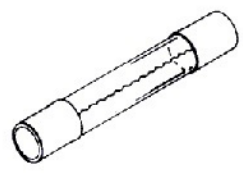

Fuse
Figure 1. Present thermal overload relays. machine will cause the relay to activate the trip circuit thereby opening the circuit by circuit breaker.

The main motive of our technique is to isolate the machine and to protect them from damage during the event of thermal overload. Also this relay has a better accuracy and shuts the power down at a specific temperature.

\section{Components}

Some of the important components used in the construction of the relay are listed below.

\subsection{Ferrofluid}

Ferrofluid was originally developed by NASA in 1960's. They are highly viscous fluids made up of nanometer sized pieces of magnetite $\left(\mathrm{Fe}_{3} \mathrm{O}_{4}\right)$ suspended in a liquid. These fluids are a special category of nano-materials which exhibit simultaneously liquid and super-paramagnetic properties. The possibility of magnetic control over their properties triggered many application orientated researches. It behaves like a ferromagnetic material in the presence of magnetic field but loses the property (behaves like paramagnetic material) in the absence of magnetic field. Also it changes its density in proportion to the strength of the magnetic field that is applied to it. However ferrofluid keeps its fluidity even if subjected to strong magnetic fields [2]. The thermal stability of this fluid depends on the type of surfactant used [3]. The 
property of ferrofluids losing their magnetic properties at a specific temperature (Curie temperature) is used in the construction of this relay. At present, ferrofluid technology is well established and capable of solving variety of technical problems.

\subsection{Magnetic Field Generator}

The ferrofluid being a magnetically active fluid, it is necessary to have a magnetic field generator to produce the required magnetic field. Relays are generally classi- fied depending on the type of magnet being used to oper- ate it. For this purpose either a permanent magnet or an electromagnet can be used. Relays using permanent magnets are known as polarized relays and those using an electromagnet are known as non polarized relays. The property of the ferrofluid greatly depends on the direction of the magnetic flux hence it is necessary to choose the perfect source of magnetic field based on the application.

\subsection{Heater Equipment}

Electrical motors require a heating element to activate the relay. In these machines the thermal overload relay is used to sense the overload current. The temperature rise of the heater is proportional to the amount of current flowing through it. Hence the selection of heating element is an important factor as it decides the current at which the relay should activate the trip circuit. Selection is based on the value of current flow during the full load operation of the machine. The ambient temperature at the motor and relay is the same in most applications. In conditions where the temperature is variable, a sensor based heater element is used.

\section{Construction}

It is very important to choose an adequate protective device for the safety of the machines during operation and for their durability. The efficiency of protection methods varies according to the application. The construction of relay plays a major part in the protection of any electrical machine. Motor terminal wire is connected to the heating element which is wound on the upper part of the container. The same heating element used in the current techniques of overload can be used in this method of thermal overload relay. A tube like cylindrical container is used to hold the ferrofluid. Magnetic field generator is placed concentrically around the container to hold the ferrofluid in place. The power supply to the generator is provided by the input terminal current of the motor. Magnetic field generator is setup in such a way that it can be moved along the vertical axis with the help of a stepper motor. This arrangement is provided to ease the process of resetting the relay once the problem is rectified.

Ferrofluid is enclosed in the upper part of the container so that it possesses a specific potential energy. Lower portion of the container consists of a movable plate connected to a spring mechanism. The spring arrangement is made to provide the necessary restraining force to keep the movable plate in position. In this relay a semi automatic reset can be used where the relay is reset by a controller based stepper motor. This method of reset is used because the ferrofluid can be activated only by the magnetic field generator which is energized by the supply current. A typical arrangement of the components for the construction of this relay is shown in Figure 2.

\section{Operation}

Thermal overload units are widely used on both the fractional and integral horsepower manual starters for protection of motors from sustained electrical over-currents [4]. This over-current could result from overloading of the driven machine or from excessively low line voltage. An electric motor does not know enough to quit when the load gets too much for it. It keeps going until it burns out [5]. If a motor is subjected over a period of time to internal or external heat levels that are high enough to destroy the insulation on the motor windings, it will fail and burn out. A solution to this problem is to install some device to protect a motor against expected overheating [6]. Hence, we use a thermal overload relay for this purpose whose current sensing properties very similar to the heating curve of the motor. The operation of the protective device is ideal if the motor is allowed to carry small, short, and harmless overloads but is quickly disconnected from the line when an overload has persisted for a long time. The motor can do no more work than the overload relay permits and provides protection against the overloads above full-load current when the motor is running. Continuous over-current through the heater unit raises

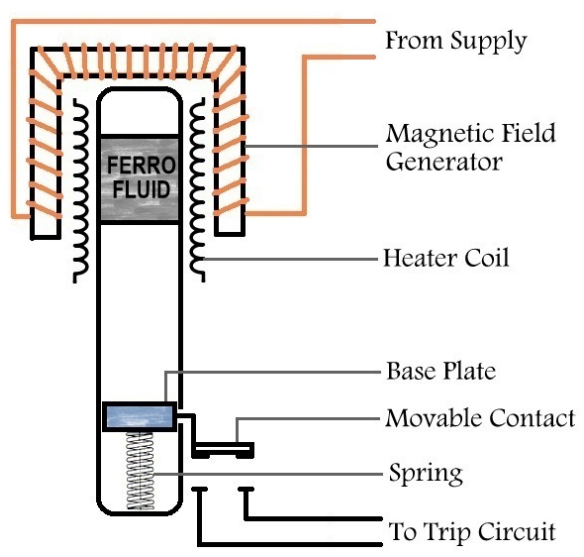

Figure 2. Construction of ferrofluid relay. 
the temperature of the heating element. Many types of overload relay heater units are available so that the proper one can be selected on the basis of the actual fullload current rating of the motor.

It is necessary to achieve an inverse time relationship with the relay operation so that normal motor starting currents and momentary overloads will not cause thermal relays to trip. The operating time is approximately inversely proportional to the magnitude of the actuating quantity. At values of current less than pickup, the relay never operates. At higher values, the time of operation of the relay decreases steadily with the increase of current. The typical inverse time characteristic of the thermal overload relay is shown in Figure 3.

In this type of relay the inverse-time delay is achieved by associating it with a heating element. The line current is given to the relay through the relay input block. The relay input block provides the supply to both the heating element and the magnetic field generator. Generally the heating element is connected in such a way that it is in series with the motor starter. The magnetic field generator will get a continuous supply from the main supply. Here a permanent magnet can also be used instead to avoid continuous power supply. The magnetic field cre- ated by the magnetic field generator holds the ferrofluid in active region.

The base plate is now at its normal position where the restraining force of the spring is greater than the weight of the plate (Operating force < Restraining force). The exact point at which the ferrofluid loses its magnetism is decided by the selection of ferrofluid (based on the curie temperature value), Magnetic field strength and the heating characteristics of the heating element. Considering a constant magnetic field strength the control is purely based on the heating element characteristic. When normal current flows through the motor, the temperature of the heating element stays below the curie temperature of the ferrofluid. As the amount of current increases and flows for a given time period, the heating element reaches the temperature value equal to the curie temperature. At this temperature the ferrofluid loses its magnetism and drops down to the base plate. The dynamics of fluid flow in a tube are dependent on a dimensionless group called the Reynolds Number [2].

$$
\text { Reynolds Number }=\mathrm{Re}=\frac{\mathrm{Ud}}{\mathrm{v}}=\frac{\text { Inertial force }}{\text { Viscous force }}
$$

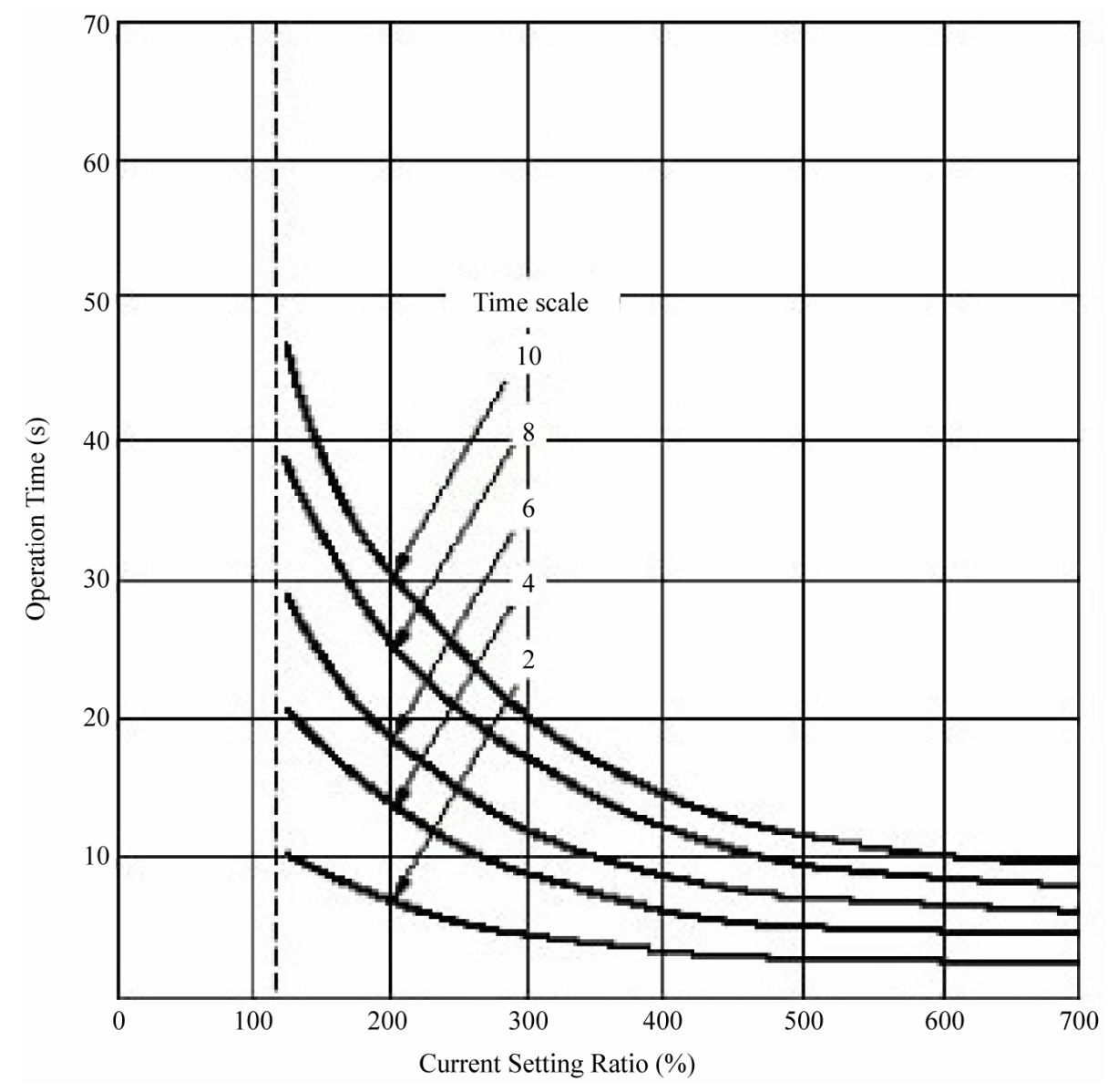

Figure 3. Inverse time characteristics during overload. 
The inertial force here is provided by the action of gravity when the magnetic property is lost. The sudden increase in weight causes the base plate to move downwards due to gravity. At this condition the weight of the base plate is more than the restraining force of the spring (Operating force $>$ Restraining force). Hence, the base plate pushes the movable contact downwards to short the fixed contact following which the trip circuit is triggered. The trip circuit eventually disconnects the motor from the supply by opening the normally closed (NC) switches. The operation of this relay can be clearly understood from Figure 4 which shows the complete working. The trip circuit is operated only during the fault condition by closing the contacts. Once the fault is cleared, the supply is given to the relay and the magnetic field generator is used to bring back the ferrofluid to its original position. This makes the movable contact to the non-operating position.

In this relay a semi automated reset mechanism is used so that once the ferrofluid is lowered it can be moved to its original position by the operation of stepper motor operated magnetic field generator. Automatic reset overload relays are not normally recommended because of the possible danger to personnel. The unexpected restarting of the machine may find the operator or electrician in a hazardous situation as attempts are made to find out why the machine has stopped.

\section{Advantages}

- Relays improve safety by providing complete electrical isolation from high current and voltages during fault in the system. It comes in all shapes and sizes for different applications and they have various switch contact configurations. As a result it can be used to switch many contacts at once.

- Thermal overload relays provide flexible protection to the motor from overheating and these relays have a

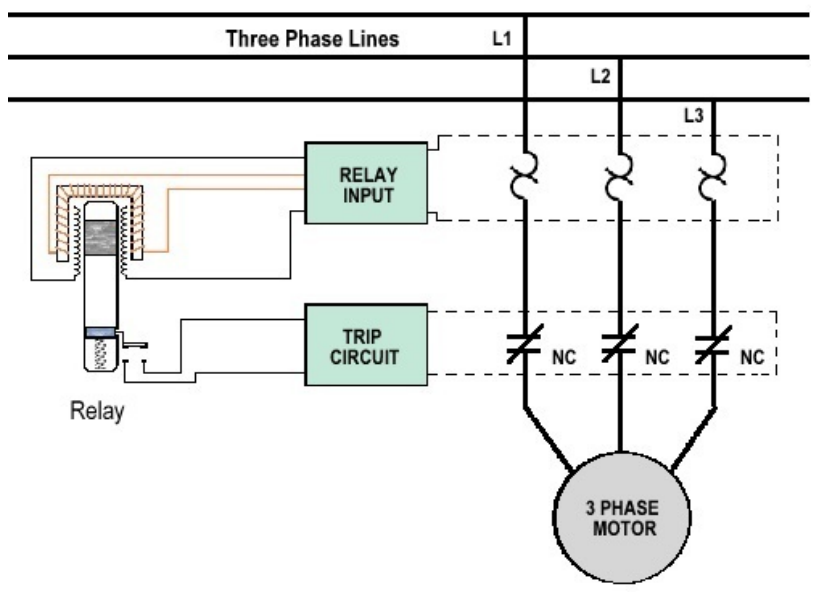

Figure 4. Operation of ferrofluid relay. tendency to resist shock and vibration of normal application. It also provide features like ambient temperature compensation (Ambient temperature compensation is an important factor as the temperature of the overload enclosure is subjected to severe fluctuation when the enclosure is expected to run either in hotter or colder temperature surrounding).

- This relay can also be used in transformer protection [7]. It is necessary to protect the transformer from overheating. The overheating of transformer will damage the transformer coil and generally the transformer temperature should be limited below $110^{\circ} \mathrm{C}$ for proper working. The relay can also be used in protecttion of generator protection. In certain cases the field winding may get overheated due to high current passing through it. Hence it is very important to protect the field winding of the generator from overheating.

- This relay has an improved accuracy as it trips the circuit at a specific temperature. The other types of overload relays are purely mechanical and dependent on the temperature characteristics of the metal being used. Here the ferrofluid relay operation has a very sharp operating characteristic as it breaks the contact when the temperature attains the curie temperature.

- It can withstand repeated trip and reset cycles without need of replacement as the property of the ferrofluid is completely reversible. Thus it is more advantageous than using a fuse which need frequent replacement after its operation.

- This ferrofluid relay also finds application in nuclear reactor. It is of high importance to limit the nuclear reactor to a specific temperature above which it may become unstable. Hence in that case this relay can be employed to slow down or shut down the operation when the temperature crosses the safety limit.

\section{Conclusions}

In this paper a new type of thermal overload relay is recommended which provides an accurate tripping of the circuit preventing the overheating of the motor. This relay is based on the property of ferrofluid which behaves like a ferromagnetic material in the presence of magnetic field but loses the property (behaves like paramagnetic material) in the absence of magnetic field. Another property of the ferrofluid used in the relay operation is that the fluid loses its magnetic property once the temperature crosses the critical temperature known as curie temperature.

When the current flowing through the motor is normal, the temperature of the heating element stays below the curie temperature of the ferrofluid thereby keeping the ferrofluid unaffected. As the amount of current flow increases and prolongs for a given time period, the heating element reaches the temperature value equal to the Curie 
temperature. At this temperature the ferrofluid loses its magnetism and drops down. The ferrofluid eventually falls on the base plate which is the operating force of the relay. This force will be greater than the restraining force of the spring which makes the circuit to trip.

Thus this new type of thermal overload relay makes tripping of circuit during overheating of the motor more accurate. It finds application in various industries and research facilities where accuracy is an important factor. Moreover in this paper the use of such smart fluid has been exposed to find its application in the electrical domain.

\section{REFERENCES}

[1] “Motor Thermal Protection,” Danaher Motion, Document
Number: A-RT-000-18 Rev 3.

[2] T. A. Franklin, "Ferrofluid Flow Phenomena," Massachusetts Institute of Technology, Cambridge, 2003.

[3] C. Scherer and A. M. F. Neto, "Ferrofluids: Properties and Applications," Brazillian Journal of Physics, Vol. 35, No. 3, 2005. doi:10.1590/S0103-97332005000400018

[4] S. L. Herman, "Electrical Motor Control," 9th Edition, Cengage Learning, Delmar, 2007.

[5] C. Bussmann, "Selecting Protective Devices Handbook," Motor Circuit Branch Circuit Protection, 2005, p. 132.

[6] Desensitizing Electric Motor Controls, Pacific Gas and Electric Company, August 2004.

[7] "Transformer Protection-The Importance of Effective Transformer and Control Transformer Protection,” Littlefuse POWR-GARD® Products, Littelfuse, 2010. 